Indexed by

\title{
THE NEW HYPOTHESIS ANGULAR DEFORMATION AND FILLING OF DIAGRAMS IN BENDING WITH TORSION IN REINFORCED CONCRETE STRUCTURES
}

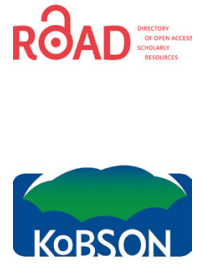

Vladimir Kolchunov

South-West State

University, Department of

Unique Buildings and

Structures, Kursk, Russian

Federation

\author{
Aleksey Dem'yanov \\ South-West State \\ University, Department of \\ Unique Buildings and \\ Structures, Kursk, Russian \\ Federation
}

\author{
Maxim Protchenko \\ South-West State \\ University, Department of \\ Unique Buildings and \\ Structures, Kursk, Russian \\ Federation
}

Key words: bending with torsion, functionals, angular deformations, bending moment, torque doi:10.5937/jaes0-32660

Cite article:

Kolchunov V., Dem'yanov A., Protchenko M. (2021) THE NEW HYPOTHESIS ANGULAR DEFORMATION AND FILLING OF DIAGRAMS IN BENDING WITH TORSION IN REINFORCED CONCRETE STRUCTURES, Journal of Applied Engineering Science, 19(4), 972-979, DOI:10.5937/ jaes0-32660

Online aceess of full paper is available at: www.engineeringscience.rs/browse-issues 


\title{
THE NEW HYPOTHESIS ANGULAR DEFORMATION AND FILLING OF DIAGRAMS IN BENDING WITH TORSION IN REINFORCED CONCRETE STRUCTURES
}

\author{
Vladimir Kolchunov*, Aleksey Dem'yanov, Maxim Protchenko \\ South-West State University, Department of Unique Buildings and Structures, Kursk, Russian Federation
}

The authors considered a simple method for constructing bend-torsion functionals by grid methods. Analysis of the diagrams of angular deformations and shear stresses made it possible to develop a new hypothesis of angular deformations. The consequences of the hypothesis were in the form of expressions from the analysis of diagrams. The authors also obtained functionals for determining angular deformations, bending and torque moments from the compressed area of concrete and reinforcement. The projection ratios helped to determine the shear and normal stresses through deformations using diagrams. The filling of the diagrams was in the form of expressions using functionals. The authors recorded expressions for determining the filling of the diagrams, as well as the total bending and torque moments.

Key words: bending with torsion, functionals, angular deformations, bending moment, torque

\section{INTRODUCTION}

Experimental and theoretical studies in the field of torsion with bending are associated with the need to develop a design scheme and take into account a number of new effects of deformation of reinforced concrete with spatial cracks. Some of the earliest research in the field of torsion with bending were presented in [1-2]. A large theoretical and experimental basis for the development of the theory of bending with torsion is described in -publications [3-6], [7-9]. The main task is to develop models that allow describing the behavior of structures at all stages of loading. Rectangular cross-sections are one of the most applicable, therefore the research of this type of cross-section is most important [10-13]. However, the complex stress state has not yet been sufficiently considered in scientific publications [14-23].

There is a problem of searching for a new hypothesis of linear and angular deformations for rectangular sections and determining the filling of curvilinear diagrams in bending with torsion. It is necessary to use the analytical functional and special functions for deplanation of the cross section to obtain practical models. Engineering proposals have not been found for projecting the coefficients of the stress-strain and elastoplastic state from the stress and strain diagram.

\section{Research methods}

Determination of deformations and stresses in a complex stress-strain state in a rectangular section can be obtained from the Timoshenko-Goodyer theory of elasticity [24] using a membrane analogy.

The function of Timoshenko and Goodyear [24] can be represented as:

$f=Y \cdot f_{*}$
Where

$Y=\frac{8 \cdot G \cdot \varphi_{A} \cdot b^{2}}{\pi^{3}}$

$\varphi_{A}-$ torsion angle for cross-section in edge fibers of compressed concrete or tensile reinforcement; $\mathrm{f}_{\mathrm{t}}$ - complex series of Timoshenko and Goodyer in the theory of elasticity. This function is complex and time consuming to calculate. Its calculation is rather difficult for plastic regions and regions with cracks. Therefore, a simple new method from the families of the mesh method was found to develop deformation functionals for approximating any rectangular mean sections in compressed and stretched zones using special squares (Fig. 1, a). A more frequent splitting of the cross-section was used with the use of other points to correct the values of the obtained function (Fig.1, b).
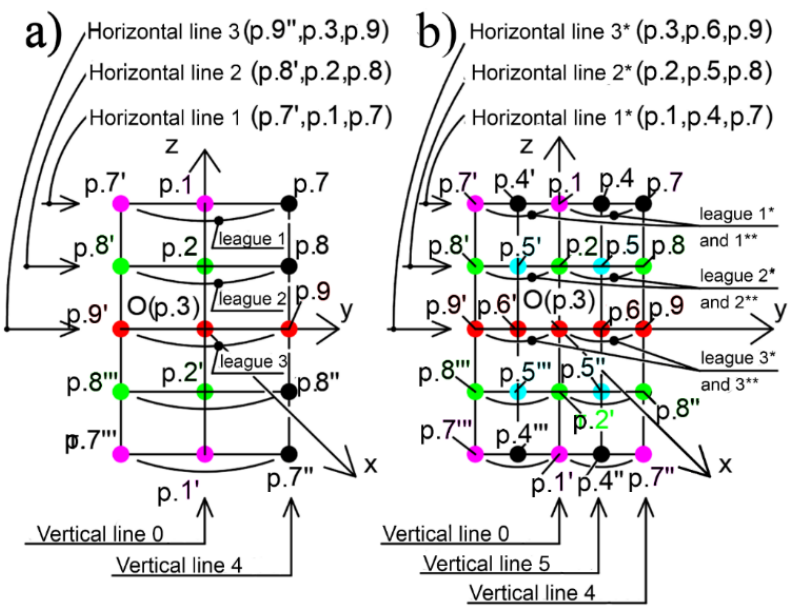

Figure 1: Approximating rectangular sections using special squares: (a) large parts of grid; (b) smaller parts of grid; leagues between points are functions along the $y$ or $z$ axes; z-leagues are not labeled for simplification 
We obtained the analytical first functional $f_{5, *}(y, z)$ after several adjustments: through function $\mathrm{f}_{1, *}(\mathrm{y})$ (horizontal parabola about the $y$-axis) and function $\mathrm{f}_{2,{ }^{*}}(\mathrm{z})$ (vertical parabola about the z-axis).

$f_{5,{ }^{*}}(y, z)=f_{1,{ }^{*}}(y) \cdot f_{2, *}(z)= \pm\left[-\frac{3\left(47 b^{2}-200 y^{2}\right.}{25 b^{2} h^{2}} \cdot z^{2}+\right.$

$\left.+\frac{487 b^{2}-2280 y^{2}}{500 b^{2} h} \cdot z+0.923-\frac{93 y^{2}}{25 b^{2}}\right]$

Where $A(y), B(y), C(y)$ - functions;

$A=-\frac{3\left(47 b^{2}-200 y^{2}\right)}{25 b^{2} h^{2}} ; B=\frac{487 b^{2}-2280 y^{2}}{500 b^{2} h}$

$C=0.923-\frac{93 y^{2}}{25 b^{2}}$

- - transition between functions; signs "+" and "-" are adopted respectively for quadrants I, III and II, IV.

We had received an error of up to $2 \%$ at the considered points and to $7 \%$ at any points of the cross section when applying our functional to find the values of the functions (Fig. 1, b).

The analytic undefined second functional is a function of three functions:

$f_{\text {*** }}(x, y, z)=e^{-3.19\left(\frac{x}{l}+\frac{3.84 h-22.96 z}{b^{2} h} \cdot(y)^{2}-\frac{2.88 h-12.3 z}{b h} \cdot y-\frac{0.34 h-0.36 z}{h}\right)}-$

$-\frac{9.39 h-27.02 z}{b^{2} h} \cdot(y)^{2}-\frac{7.16 h-17.39 z}{b h} \cdot y-\frac{-0.306 h+0.232 z}{h}$

The error is $15 \%$ for the first iteration and $2 \%$ for the second iteration.

We have developed a new hypothesis and formulated the definition. The proposed new hypothesis of angular deformations - the kinematics between fibers for the relative transverse fiber upper and lower total shear strains of concrete and reinforcement $\left(\gamma_{\text {sum,b }}\right.$ and $\left.\gamma_{\text {sum }, s}\right)$ to determine their ratios in distances from the neutral axis, which has a special geometric figure for the function $\mathrm{f}_{\text {sum, } y}$ (signs "+", "-" taken for different quadrants), as well as the parameter between concrete in plastic and elastic areas to obtain an equation with deformation $\mathrm{f}_{\mathrm{b}, \mathrm{el}}$.

Note: there is a special section 3-3, where the local corner regions do not have a kinematic connection between the outermost fibers through the neutral axis of the section.

We have determined the corollaries of the hypothesis. Corollary 1 . The proportion for a trapezoid (section 2-2 for $y=b / 8, b / 4,3 b / 8$ ) and a triangle (section $1-1$, ) has the form (Fig. 2):

$$
\frac{r_{2}}{r_{1}}=\frac{V_{2} \cdot \varphi_{\text {los }}}{V_{1}}
$$

$\varphi_{\text {los }}$ - coefficient from to zero in the form of a parabola:

$\varphi_{\text {los }}=A \cdot y^{2}+B \cdot y+C$

From point $1\left(\mathrm{~V} \cdot \mathrm{Y}_{\max } ; 0\right)$, point $2(0 ; 0.5 \mathrm{~b})$ and point $3(0$; $-0.5 b)$ we got:

$C=\gamma \cdot V_{\max }$

$$
\begin{aligned}
& A=\frac{-\gamma \cdot V_{\text {max }}}{(0,5 b)^{2}} \\
& B=\frac{-\gamma \cdot \gamma_{\text {max }}+\gamma \cdot \gamma_{\text {max }}}{0.5 b}=0 \\
& \varphi_{\text {los }}=\gamma \cdot V_{\text {max }}-\frac{\gamma \cdot \gamma_{\text {max }}}{(0,5 b)^{2}} \cdot y^{2}
\end{aligned}
$$

Corollary 2. Reduction of the zone of compressed concrete from the load (Fig. 2) has the form:

$x_{k, *}=\frac{x_{k}\left(h_{0}-x_{k,{ }^{*}}\right)}{h_{0}-x_{k}}$

Corollary 3. The coefficients $Y_{3}$ and $Y_{4}$ were found from two pairs of triangles:

$\frac{V_{2}\left(z_{k^{*}}+\left(x_{k}-x_{k, *}\right)\right)}{V_{4} \cdot z_{k^{*}}}=\frac{V_{3}\left(z_{k}-\left(x_{k}-x_{k, *}\right)\right)}{V_{2} \cdot z_{k}}$

$\gamma_{2}+\gamma_{2}=\gamma_{3}+\gamma_{4}$

The distances $z_{k}$ and $z_{k^{*}}$ were found:

$z_{k}=z_{k^{*}}+x_{k}-x_{k, *}$

$\frac{z_{k, *}^{*}}{V_{4}}=\frac{z_{k}+x_{k}-x_{k, *}}{V_{3}}$

$z_{k, *}=\frac{V_{4}\left(x_{k}-x_{k, *}\right)}{V_{2}-V_{4}}$

$z_{k}=\frac{\left(x_{k}-x_{k, *}\right) \gamma_{2}}{\gamma_{2}-\gamma_{4}}$

The coefficient $\gamma_{3}$ was expressed from the equations (10) and (11):

$V_{3}=\frac{V_{2}^{4}}{V_{4}^{3}}$

We got after algebraic transformations in equation (12):

$\gamma_{4}{ }^{4}-\gamma_{4}{ }^{3} \cdot 2 \gamma_{2}+\gamma_{2}{ }^{4}=0$

The equation (18) can be solved by iterating.

Thus, the corollary of the hypothesis has the form:

Corollary 1. The proportion for a trapezoid of angular deformations is the ratio of a vector $r_{1}$ to any point $A$ and its horizontal projection $r_{2}$. The proportion is used in a trapezoid (section 2-2, $y=b / 8, b / 4,3 b / 8$ ), in a triangle (section $\left.1-1, y=0, r_{2}=0\right)$, but is not used in a special section 3-3 $(y=0,5 b)$. The coefficient $\varphi_{\text {los }}$ was obtained in the form of a parabola (9).

Corollary 2. The decrease in the distance from the neutral axis of the compressed concrete under load is the proportion (10).

Corollary 3. Angular deformations $\mathrm{Y}_{3}$ and $\mathrm{Y}_{4}(17)$, (18)) with decreasing distance from the neutral axis of compressed concrete can be found from the geometric proportions (11), (12) in Fig. 2,c.

The component of the relative angular deformations $Y_{t, z x}$ 


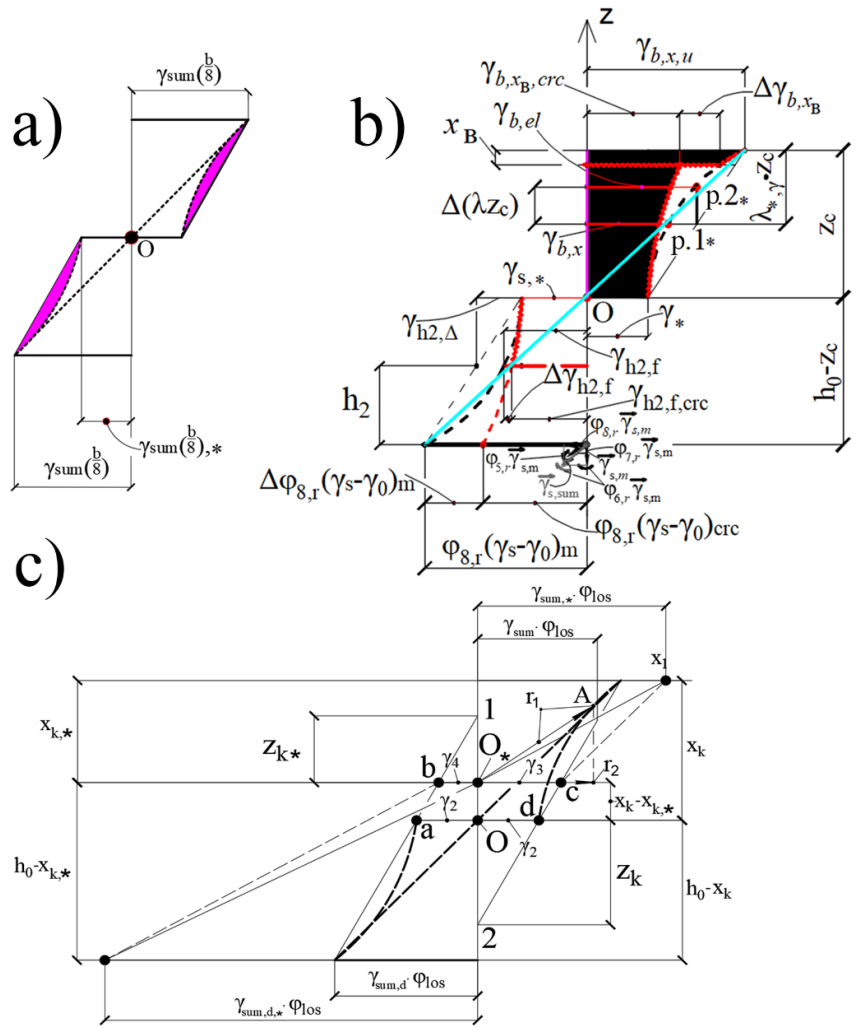

Figure 2: Diagram of the shear strain of concrete and reinforcement in section 2-2: for stage I (a), for stage II (b), parameters from the corollary of the angular deformations hypothesis (c)

was obtained from the undefined functional $f_{5,}(y, z)$ obtained above using differentiation:

$V_{t, z x}=\frac{Y}{G(\lambda)} \cdot\left(-\frac{d f_{5, *}}{d y}\right)=\frac{8 \cdot \varphi_{A} \cdot b^{2}}{\pi^{3}} \cdot\left(-\frac{d f_{5, *}}{d y}\right)=$

$=\frac{8 \cdot y \cdot \varphi_{A}}{25 b^{2} h^{2} \pi^{3}} \cdot\left(186 h^{2}+228 h z-1200 z^{2}\right)$

Where $\mathrm{f}_{5, \mathrm{t}}$-functional in (2);

$Y=\frac{8 \cdot G(\lambda) \cdot \varphi_{A} \cdot b^{2}}{\pi^{3}}$

$\varphi_{A}$ - torsion angle for cross-section in edge fibers of compressed concrete or tensile reinforcement.

The component of the relative angular deformations $\gamma_{t, y x}$ was obtained in a similar way.

$Y_{t, z x}=\frac{Y}{G(\lambda)} \cdot \frac{d f_{5, *}}{d z}=\frac{8 \cdot \varphi_{A} \cdot b^{2}}{\pi^{3}} \cdot \frac{d f_{5, *}}{d z}=$

$=\frac{2 \cdot\left(487 b^{2}-2280 y^{2}\right) \cdot \varphi_{A}}{125 h \pi^{3}}-\frac{48 \cdot\left(47 b^{2}-200 y^{2}\right) \cdot z \cdot \varphi_{A}}{25 h^{2} \pi^{3}}$

The total shear deformations have the form:

$V_{\text {sum }, V}=\sqrt{V_{t, z x}^{2}+V_{t, y x}^{2}}=$

$=\frac{2 \varphi_{A}}{125 h^{2} \pi^{3}} \cdot\left[\left(b^{2}(487 h-5640 \cdot z)+16 y^{2}(-101 h+1320 z)\right)^{2}+\right.$

$\left.+256 y^{2}\left(229 h^{2}+202 h z-1320 z^{2}\right)^{2}\right]^{\frac{1}{2}}$
When passing from plastic to elastoplastic deformation at point 2, we get:

$f_{\text {sum }, y}(p .2)=\gamma_{\text {sum }, e l}=\sqrt{\gamma_{z x, e l}^{2}+\gamma_{y x, e l}^{2}}=$

$\frac{2 \varphi_{A}}{125 h^{2} \pi^{3}} \cdot\left[\left(b^{2}\left(487 h-5640 \cdot\left(z_{c}-\lambda_{t, V} \cdot z_{c}\right)\right)+\right.\right.$

$\left.+16 y^{2}\left(-101 h+1320 \cdot\left(z_{c}-\lambda_{t, V} \cdot z_{c}\right)\right)\right)^{2}+$

$\left.256 y^{2}\left(229 h^{2}+202 h \cdot\left(z_{c}-\lambda_{t, r} \cdot z_{c}\right)-1320 \cdot\left(z_{c}-\lambda_{t, r} \cdot z_{c}\right)^{2}\right)^{2}\right]^{\frac{1}{2}}$

Shear strains from torsion have jumps in the diagram (Fig. 2, b). The deformation function during crack formation $\mathrm{f}_{\text {sum. } ., \Delta 1 \text { crc }}$ (jump 1 on the deformation diagram) is similar to the function from formula (22), only less by a coefficient $\mathrm{k}_{\mathrm{v}, \mathrm{sum}}$ :

$f_{\text {sum }, \gamma, \Delta, \text { crc }}=\frac{f_{\text {sum }, \gamma}}{k_{y, \text { sum }}}=$

$=\frac{1}{k_{\mathrm{y}, \text { sum }}} \frac{2 \varphi_{A}}{125 h^{2} \pi^{3}} \cdot\left(b^{2}\left((487 h-5640)+16 y^{2}(-101 h+1320)\right)^{2}+\right.$

$+256 y^{2}\left(229 h^{3}+202 h z-1320 z^{2}\right)^{2}$

Where $f_{\text {sum }, \mathrm{v}}-a$ function for calculating shear strains without cracks (22); $f_{\text {sum, }, \Delta, \Delta 1, \text { crc }}-$ a function for calculating shear deformations in a section with one spatial crack at the end of the upper compressed region $\mathrm{x}_{\mathrm{B}}$.

The function for the second jump during crack formation has the form:

$f_{v, \Delta_{2}}=f_{\text {sum }, \gamma}-f_{\text {sum }, \gamma, A_{1}, \text { crc }}=f_{\text {sum }, \gamma}-\frac{f_{\text {sum }, \gamma}}{k_{\gamma, \text { sum }}}=f_{\text {sum }, \gamma}\left(1-\frac{1}{k_{\gamma, \text { sum }}}\right)$

The function of deformations at the appearance of the second crack-trace has the form:

$f_{\text {sum },,, Q_{2}, t r}=f_{\text {sum, }, Q_{1}, \text { crc }}-f_{\Delta Y}=$

$=\frac{f_{\text {sum }, V}}{k_{\gamma, \text { sum }}}-f_{\text {sum }, \gamma}+\frac{f_{\text {sum }, \gamma}}{k_{\gamma, \text { sum }}}=f_{\text {sum }, \gamma}\left(\frac{2}{k_{\gamma, \text { sum }}}-1\right)$

When analyzing with the approximation of the branches of the graph of the Timoshenko-Goodyer function [24] and the first functional, we obtain an error of up to $7 \%$ (Fig. 3, a) and less than 1\% (for Fig. 3, b).

We also get the coefficients for projecting normal and shear stresses using deformations from the diagrams

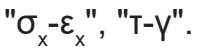

When analyzing with the approximation of the branches of the graph of the Timoshenko-Goodyer function [24] and the first functional, we obtain an error of up to $7 \%$ (Fig. 3, a) and less than 1\% (for Fig. 3, b).

The authors obtained the coefficients and from points $C$, $B, A, D$ using the projection of deformations and stresses for the Prandtl diagram with constraints: $j=C, B, A, D$; $\sigma_{x}=\varepsilon_{x} \cdot E_{b}(\lambda)=\varepsilon_{x} \cdot v(\lambda) \cdot E_{b} ;$

$E_{b, j}(\lambda)=\frac{\sigma_{b, i}}{\varepsilon_{b, i}}$ for point A- $E_{b, e l}=\frac{\sigma_{i, e l}}{\varepsilon_{i, e l}}=\frac{R_{b}}{0,0015}$ 
a)

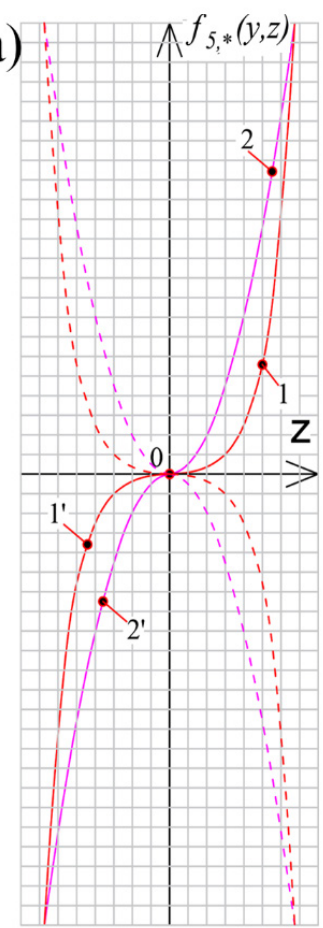

Figure 3: Approximation of branches of graphs of

Timoshenko-Goodyère functions (1) and our new first functional (2): in axes $f_{5,}(y, z)-z(a)$, in axes $f_{5, n, *}(y, z)-y(b)$

$\mu_{b, e l}=0,167$ for point B- $E_{b R}(\lambda)=\frac{\sigma_{i, b R}}{\varepsilon_{i, b R}}=\frac{R_{b}}{0.0020}$

$\mu_{b R}(\lambda)=0.251$ for point C- $E_{b, u}(\lambda)=\frac{\sigma_{i, u}}{\varepsilon_{i, u}}=\frac{R_{b}}{0.0035}$

$\mu_{b, u}(\lambda)=0,357$ for point $F_{k}$

$\operatorname{tg} \alpha_{F_{k}}=\frac{\sigma_{x, u}}{\varepsilon_{x, F_{k}}}=E_{b, F_{k}}(\lambda)=E_{b} v_{b, F_{k}}(\lambda), v_{b, F_{k}}(\lambda)=\frac{\sigma_{x, u}}{\varepsilon_{x, F_{k}} \cdot E_{b}}$

The coefficients $\varphi_{\mathrm{ij}}$ for parameters with limited angular deformations at point $\mathrm{C}$ have the form:

$\varphi_{\gamma_{z x}, u}=\frac{V_{z x, u}}{\varepsilon_{1, u}}=\frac{\left(\varepsilon_{x_{1}, u}-\varepsilon_{z_{1}, u}\right) \cdot \sin 2 \alpha+\gamma_{z_{1} x_{1}, u} \cos 2 \alpha}{1,567 \cdot \varepsilon_{i, u}+\varepsilon_{3, u}}=$

$=\frac{\varepsilon_{x_{1}, u}-\varepsilon_{z_{1}, u}}{1,567 \cdot \varepsilon_{i, u}+\varepsilon_{3, u}} \leq \frac{T_{p l}\left(\frac{c}{h_{0}}\right) \cdot 2\left(1+\mu_{b, u}(\lambda)\right)}{E_{b, u}(\lambda) \cdot\left(1,567 \cdot \varepsilon_{i, u}+\varepsilon_{3, u}\right)}$

Where $\alpha$-angle between cross section and inclined section. For the coefficient $\varphi_{\mathrm{yy} x, \mathrm{u}}$, we get:

$\varphi_{Y_{y x}, u}=\frac{V_{y x, u}}{\varepsilon_{1, u}}=\frac{k_{x, u} \cdot k_{x *} \cdot Y_{z x, M t, e l}}{1,567 \cdot \varepsilon_{i, u}+\varepsilon_{3, u}}$

The third undefined functional for bending moment is obtained by integrating the special function $\mathrm{f}_{\text {sum }, \Delta-\mathrm{d}}$ :

$f_{\varepsilon, \text { int,vol }}(x, y, z)=\iiint f_{\text {sum }, \Delta-d} d x d y d z=\iiint \varepsilon_{x, \text { sum }} d x d y d z$

Where

$f_{\text {sum }, V-d}= \pm\left[B_{1} \cdot\left(z-z_{c}\right)+B_{2} \cdot\left(h_{0}+z-z_{c}\right)\right] \cdot B_{3} \cdot B_{4} \cdot x \pm$ $\pm D_{1} \cdot y \cdot z\left[-D_{2} \cdot x \cdot e^{-\lambda \ldots\left(\frac{x}{l}+A_{\ldots}\right)}+D_{3} \cdot e^{-\lambda \ldots\left(\frac{x}{l}+A_{\ldots}\right)}+D_{4}\right]$

parameters: $B_{1}=\frac{\varepsilon_{s, m}}{h_{0}} \quad B_{2}=\frac{\varepsilon_{b, u}}{h_{0}} \quad B_{4}=\left[\varepsilon_{b, x}\right]_{1}=\frac{R_{s u p} \cdot 1}{E_{b} \cdot v_{b} \cdot \omega_{\varepsilon} \cdot z_{c}}$

$D_{1}=\frac{M_{t}}{G_{\text {rec }} \cdot I_{\text {rec }}} \cdot \frac{a_{*}^{2}-b_{*}^{2}}{a_{*}^{2}+b_{*}^{2}} \quad D_{2}=\frac{\lambda_{* * *}}{l^{2}} \quad D_{3}=\frac{1}{l} \quad D_{4}=\frac{C_{* * * t}}{l}$

$C_{\ldots . .}(y, z)=\frac{9.39 h-27.02 z}{b^{2} h} \cdot(y)^{2}-\frac{7.16 h-17.39 z}{b h} \cdot y-\frac{-0.306 h+0.232 z}{h}$

$a_{*}=\frac{h}{2} \quad b_{*}=\frac{b}{2}$

The third definite functional before the formation of cracks has the form:

$f_{\varepsilon, \text { def }, \text { int, vol }}^{*}(x, y, z)=\left.\left[\left.\left[\left.\left[f_{\varepsilon, \text { int, vol }}(x, y, z)\right]\right|_{-0.5 h} ^{0.5 h}\right]\right|_{-0.5 b} ^{0.5 b}\right]\right|_{0} ^{a-s \cdot c}$

Third defined functional after cracking:

$f_{\varepsilon, \text { defint,vol }}^{\prime \prime}(x, y, z)=$

$=\int_{0}^{x-x_{B}} \int_{-0.5 b}^{0.5 b} \int_{0}^{a-s . c}\left(f_{\text {sum }, \Delta-d}-f_{\text {sum }, \Delta, \Delta, \Delta-d, \text { crc }}\right) d x d y d z+$

$+\int_{x-x_{B}-0.5 b}^{x} \int_{0}^{0.5 b} \int_{0}^{a-s-c}\left(f_{\text {sum }, \Delta-d}-f_{\text {sum }, \Delta, \Delta-d, d, c r c}\right) d x d y d z$

Where $\alpha$ - the distance from the support to the force, - a spatial crack for projection onto the horizontal axis, - segments from $1 / 6 \cdot c$ to six cross-sections);

$f_{\text {sum }, \Delta-d, \Delta_{1,0 c}}$ - special jump function on diagram of linear deformation. The indefinite fourth functional for the torque is obtained after integrating function $f_{5,}(y, z)(2)$ :

$f_{5, \ldots, \text {, }}(z, y)=2 \int_{0}^{y} \int_{0}^{z} f_{5, *} d y d z=$

$=2 \int_{0}^{y} \int_{0}^{z}\left[A(y) \cdot z^{2}+B(y) \cdot z+C(y)\right] d y d z=$

$=A_{\iint} \cdot \frac{2}{3} z^{3}+B_{\iint} \cdot z^{2}+C_{\iint} \cdot 2 z$

The functions $A_{\iint} B_{\iint} C_{\iint}$ take the form:

$A_{\iint}=\int_{0}^{y} A_{i j} d y=-\frac{141 y}{25 h^{2}}+\frac{8 y^{3}}{b^{2} h^{2}}$

$B_{\iint}=\int_{0}^{y} B_{i j} d y=\frac{487 y}{500 h}-\frac{760 y^{3}}{500 b^{2} h}$

$C_{\iint}=\int_{0}^{y} C_{i j} d y=\frac{923 y}{1000}-\frac{458 y^{3}}{375 b^{2}}$

The indeterminate torque $M_{t, i}=(z, y)$ can be represented as a function of the torsion angle $\varphi_{\mathrm{A}, \mathrm{i}}(\mathrm{z}, \mathrm{y})$ :

$M_{t, i}(z, y)=Y_{2} \cdot \varphi_{A, i}(z, y) \cdot f_{5, \ldots, \text {,j }}(z, y)=$

$=\frac{8 \cdot G(\lambda) \cdot b^{2}}{\pi^{3}} \cdot \varphi_{A, i}(z, y) \cdot 2 \int_{0}^{y} \int_{0}^{z} f_{5, *} d y d z=$

$=\frac{8 \cdot G(\lambda) \cdot b^{2}}{\pi^{3}} \cdot \varphi_{A, i}(z, y) \cdot\left(A_{\iint} \cdot \frac{2}{3} z^{3}+B_{\iint} \cdot z^{2}+C_{\iint} \cdot 2 z\right)$

Istraživanja i projektovanja za privredu ISSN 1451-4117 Journal of Applied Engineering Science Vol. 19, No. 4, 2021 
Where $Y_{2}=\frac{8 \cdot G(\lambda) \cdot b^{2}}{\pi^{3}} f_{5, *}(Z, y)-$ functional (2).

The torsion angle $\varphi_{A, i}$ for each point $A_{i}$ of the cross-section has the form:

$$
\begin{aligned}
& \varphi_{A, i}(z, y)=\frac{M_{t, i}(z, y) \cdot \pi^{3}}{8 \cdot G(\lambda) \cdot b^{2} \cdot f_{5, \ldots, j}(z, y)}= \\
& =\frac{M_{t, i}(z, y) \cdot \pi^{3}}{8 \cdot G(\lambda) \cdot b^{2} \cdot\left(A_{\iint} \cdot \frac{2}{3} z^{3}+B_{\iint} \cdot z^{2}+C_{\iint} \cdot 2 z\right)}
\end{aligned}
$$

Where

$$
M_{t, i}(z, y)=\gamma_{t, \text { sum }} \cdot \omega_{\gamma_{t s \text { sum }}} \cdot z_{b, i} \cdot A_{b, i}
$$

or

$$
M_{t, s, i}(z, y)=\gamma_{t, s, \text { sum }} \cdot \omega_{\gamma, s_{t, s, s u m}} \cdot z_{s, i} \cdot A_{s, i}
$$

The definite fourth functional for the torque is obtained after integrating function $f_{5, *}(y, z)(2)$ :

$$
M_{t}=Y \cdot f_{5, \iint}=\frac{8 \cdot G(\lambda) \cdot \varphi_{A} \cdot b^{2}}{\pi^{3}} \cdot 2 \int_{-0,5 b}^{0.5 b} \int_{-0.5 h}^{0.5 h} f_{5, *}(y, z) d y d z=
$$$$
=Y \cdot 0.628 \cdot b h
$$

Where $f_{5, *}(y, z)$ - functional $(2)$;

$$
\begin{aligned}
& Y=\frac{8 \cdot G(\lambda) \cdot \varphi_{A} \cdot b^{2}}{\pi^{3}}=\frac{M_{t}}{0.628 \cdot b h} \\
& f_{5, \iint}=2 \int_{-0,5 b}^{0.5 b} \int_{-0.5 h}^{0.5 h} f_{5, *}(y, z) d y d z=0.628 \cdot b h
\end{aligned}
$$

\section{RESULTS}

The bending and torque moments for deformation or stresses were determined, as well as the filling area of the deformation and stress diagrams.

The indefinite bending moment and the definite bending moment for the small square are of the form:

$M_{\text {bend, }, i}=v_{b, i}(\lambda) \cdot E_{b} \cdot A_{b, c, i}(z) \cdot z_{b, \varepsilon, i}(z) \cdot f_{\varepsilon,-\infty \text { int, vol,i }}(x, y, z)=$

$=Y_{\text {bend,i }} \cdot l_{l_{, i}}(x, y, z)=\varepsilon_{x, i}(x, y, z) \cdot v_{b, i}(\lambda) \cdot E_{b} \cdot \omega_{\varepsilon, i}(x, y, z) \cdot A_{b, c, i}(z) \cdot z_{b, e, i}(z)=$

$=\sigma_{b, x, i} \cdot \omega_{\sigma, i}(x, y, z) \cdot A_{b, c, i}(z) \cdot z_{b, \sigma, i}(z)$

and

$M_{\text {bend,def,i }}=\left.v_{b}(\lambda) \cdot E_{b} \cdot A_{b, c, i} \cdot z_{b, i} \cdot\left[\left.\left[\left.\left[f_{\varepsilon, \text { int,vol }}^{\prime \prime}(x, y, z)\right]\right|_{a_{n}} ^{a_{n+1}}\right]\right|_{b_{n}} ^{b_{n+1}}\right]\right|_{h_{n}} ^{h_{n+1}}=$

$=Y_{b e n d, c, i} \cdot l_{*, i}=\varepsilon_{x, i} \cdot v_{b, i}(\lambda) \cdot E_{b} \cdot \omega_{\varepsilon, i} \cdot A_{b, c, i} \cdot z_{b, \varepsilon, i}=$

$=\sigma_{b, x, u} \cdot \omega_{\sigma, i} \cdot A_{b, c, i} \cdot z_{b, \sigma, i}$

Where $\mathrm{v}_{\mathrm{b}, \mathrm{i}}(\lambda)$ - elastoplastic coefficient;

$Y_{\text {bend }, i}=E_{i}(\lambda) \cdot \frac{1}{\rho_{A, i}}=E_{i}(\lambda) \cdot \frac{\varepsilon_{x, \max , i}}{Z_{c, i}}$

$\omega_{\varepsilon, i}(x, y, z) ;\left(\omega_{\sigma, i}(x, y, z)\right)-$ the filling area of the linear deformation (or normal stress) diagram for a small square;

$I_{*, i}(x, y, z)=A_{b, c, i} \cdot z_{b, \varepsilon, i} \cdot f_{\varepsilon,-\circ \text { int,vol,i }}(x, y, z) \cdot \rho_{A, i}=$

$=A_{b, c, i} \cdot z_{b, \varepsilon, i} \cdot f_{\varepsilon,-\circ \text { int,vol,i }}(x, y, z) \frac{z_{c, i}}{\varepsilon_{x, i}}$ $\mathrm{z}_{\mathrm{b}, \mathrm{f}, \mathrm{i}} ;\left(\mathrm{z}_{\mathrm{b}, \mathrm{f}, \mathrm{i}}(\mathrm{z})\right)$ - distance from a point $\mathrm{A}_{\mathrm{i}}$ to the neutral axis of the cross section; $A_{b, c, i}(z)$ - small square area;

$I_{*, i}=A_{b, c, i} \cdot z_{b, \varepsilon, i} \cdot f_{\varepsilon,-\infty}$ int,vol,i$\cdot \rho_{A, i}=A_{b, c, i} \cdot z_{b, \varepsilon, i} \cdot f_{\varepsilon,-\infty \text { int }, v o l, i} \frac{z_{c, i}}{\varepsilon_{x, i}}$

$Y_{\text {bend }, c, i}=E_{i}\left(\lambda_{c}\right) \cdot \frac{1}{\rho_{A}}=E\left(\lambda_{c}\right) \cdot \frac{\varepsilon_{x, \max }}{Z_{c}}$

$\omega_{\varepsilon, j} ;\left(\omega_{\sigma, i}\right)$ - numerical value of the filling area of the deformation (stress) diagram for a small square; $\mathrm{z}_{\mathrm{b}, \mathrm{i}, \mathrm{i}}\left(\mathrm{z}_{\mathrm{b}, \mathrm{\sigma}, \mathrm{i}}\right)-\mathrm{nu}$ merical value of distance; $A_{b, c, i}$ - numerical value of area. The filling area of the diagram $\omega_{\varepsilon, \text { def }, i}$ and the distance $\mathrm{z}_{\mathrm{b}, \mathrm{i}}$ from point $\mathrm{A}_{\mathrm{i}}$ to the neutral axis for indefinite bending moment have the form:

$\omega_{\varepsilon, \text { def }, i}=\frac{\int_{a_{n}}^{a_{n+1}} \int_{b_{n}}^{b_{n+1}} \int_{h_{n}}^{h_{n+1}} f_{\text {sum }, \mathrm{v}-d} d x d y d z}{\varepsilon_{b, x, i} \cdot A_{b, c, i}}$

and

$$
\begin{aligned}
& z_{b, i}=z_{c} \frac{\sum_{i=1}^{i} S_{i}}{\sum_{i=1}^{j} A_{i}}= \\
& =z_{c}-\frac{\sum_{i=1}^{i}\left(\lambda_{t} \cdot z_{c} \cdot b \cdot\left(z_{c}-0.5 \cdot \lambda_{t} \cdot z_{c}\right)+0.5 \cdot\left(z_{c}-\lambda_{t} \cdot z_{c}\right) \cdot b \cdot \frac{2}{3}\left(z_{c}-\lambda_{t} \cdot z_{c}\right)\right)_{i}}{\sum_{j=1}^{j}\left(\lambda_{t} \cdot z_{c} \cdot b+0.5 \cdot\left(z_{c}-\lambda_{t} \cdot z_{c}\right) \cdot b\right)_{i}}
\end{aligned}
$$

Where $S_{i}$ - moment of area; $A_{i}$ - small square area.

The filling area $\omega_{\varepsilon, j}(x, y, z)$ of the diagram and the distance $\mathrm{z}_{\mathrm{b}, \mathrm{i}}$ for indefinite bending moment have a similar shape.

The indefinite torque and the definite torque for the small square are of the form:

$$
\begin{aligned}
& M_{t, i}(z, y)=Y_{2} \cdot \varphi_{A, i}(z, y) \cdot f_{5, \ldots, j]}(z, y)=Y_{*, i}(z, y) \cdot I_{t, i}(z, y)= \\
& =Y_{t, s u m, i} \cdot v_{b, i} \cdot G_{b, i} \cdot \omega_{y, i}(y, z) \cdot z_{b, y, i}(z) \cdot A_{b, i}(z)= \\
& =T_{t, s u m, i} \cdot \omega_{t_{i, i}}(y, z) \cdot z_{b, r, i}(z) \cdot A_{b, i}(z)
\end{aligned}
$$

and

$M_{t, d e f, i}=Y_{2} \cdot \varphi_{A, i} \cdot f_{5, \ldots, \text { If }}=Y_{*, i} \cdot I_{t, i}=$

$=Y_{t, s u m, i} \cdot v_{b, i} \cdot G_{b, i} \cdot \omega_{\gamma, d e f, i} \cdot z_{b, y, i} \cdot A_{b, i}=$

$=T_{t, \text { sum }, i} \cdot \omega_{r_{t} \text { defi, }} \cdot z_{b, r, i} \cdot A_{b, i}=$

$=\left(y_{t, b, s u m, i} \cdot \lambda_{t, v} \cdot z_{c}\right) \cdot\left(z_{c}-0,5 \cdot \lambda_{t, v} \cdot z_{c}\right) \cdot b+$

$+0,5 \cdot \gamma_{t, b, \text { sum }} \cdot\left(z_{c}-\lambda_{t, v} \cdot z_{c}\right) \frac{2}{3}\left(z_{c}-\lambda_{t, v} \cdot z_{c}\right) \cdot b$

Where $G_{b, i}$ - shear modulus for a small square; $v_{b, i}$ elastoplastic coefficient;

$I_{t, i}(y, z)=\frac{8 \cdot b^{2}}{\pi^{3}} \cdot f_{5, \iint}(z, y) \cdot l \quad Y_{*, i}(z, y)=G(\lambda) \cdot \varphi_{A, i}(z, y) \cdot \frac{1}{l^{2}}$

$\omega_{y, i}(y, z)\left(\omega_{T_{t} i}(y, z)\right)$ - filling area of the diagram of angular deformations (shear stresses); $A_{b, c, i}(z)$ - small square area; $\omega_{\gamma, \text { def } i}\left(\omega_{\tau_{t} \text { def } i}\right)$ - numerical value of the filling area of the angular deformations (shear stresses) diagram for a 


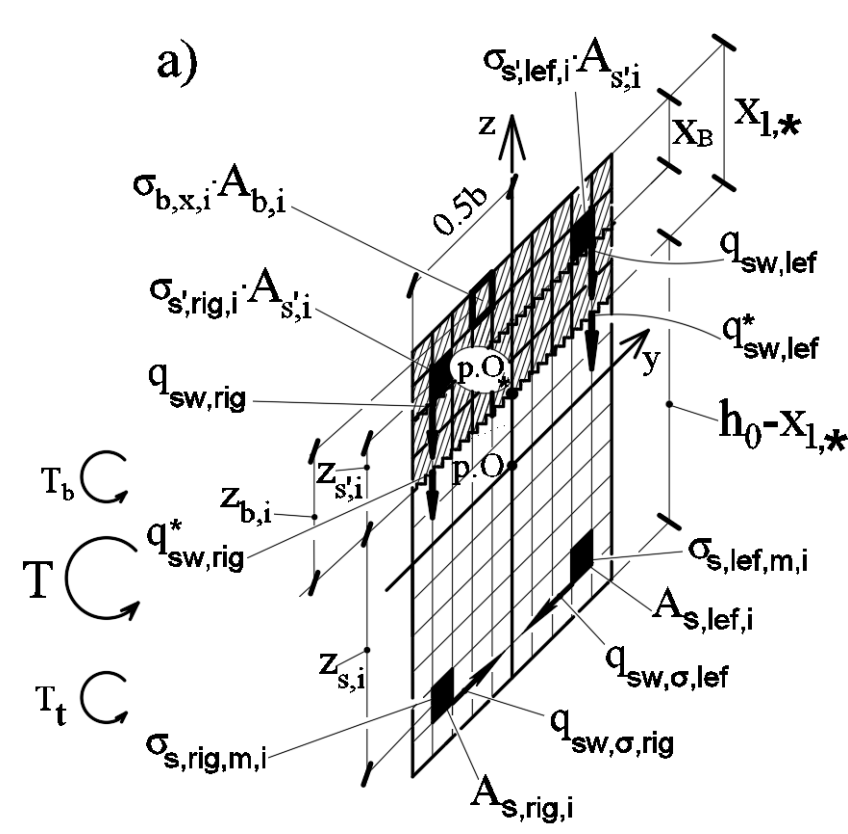

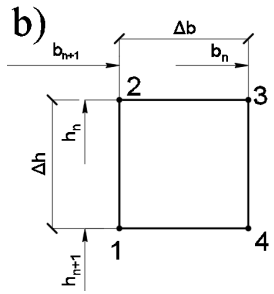
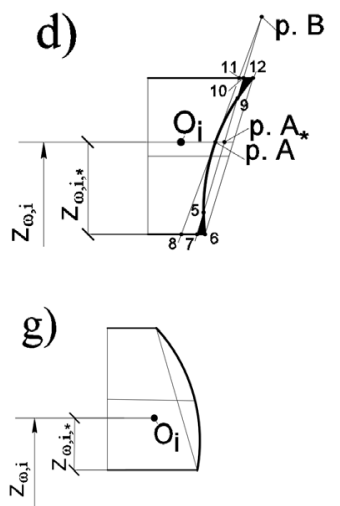
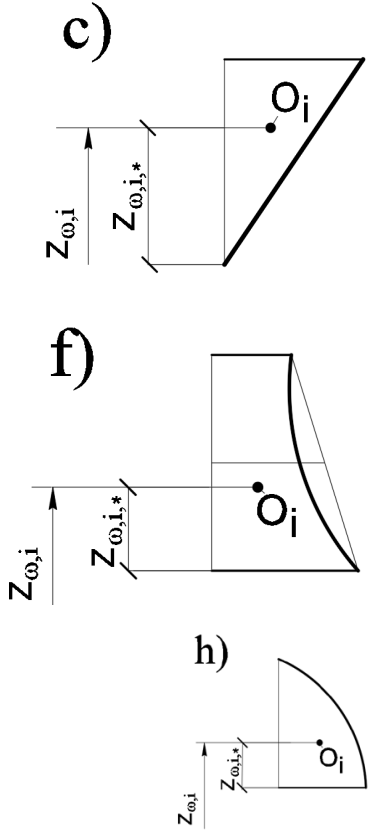

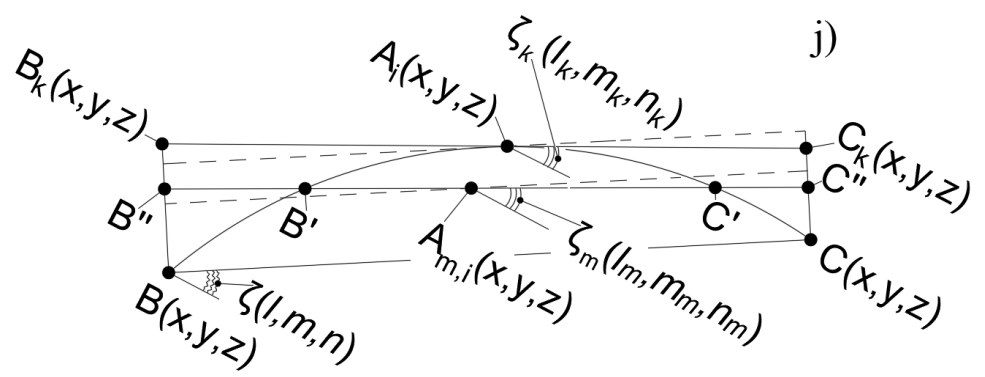

Figure 4: Small square approximation (a) in compressed concrete and reinforcement for bending and torque moments: in the form of a curved trapezoid and other shapes (b-h); straight line for angles $\zeta(I, m, n)$ or angles at apex $\zeta_{k}$ or mean angles $\zeta_{m}(j)$

small square; $z_{b, y, i}(z)\left(z_{b, T, i}(z)\right)$ - distance from a point $A_{i}$ to the neutral axis of the cross section.

The filling area of the diagram $\omega_{\mathrm{\gamma}, \mathrm{i}}(\mathrm{y}, \mathrm{z})$ have the form:

$\omega_{\gamma, i}=\frac{Y_{2} \cdot \varphi_{A, i}(z, y) \cdot f_{5,}, \int j(z, y)}{\gamma_{t, b, s u m, u} \cdot v(\lambda) \cdot G_{b} \cdot A_{b, c, i} \cdot z_{b, t, i}}$

The filling area $\omega_{y, i}(x, y, z)$ of the diagram and the distance $z_{b, i}$ for indefinite torque have a similar shape.

We have elastic, plastic regions and cracks (lateral, normal, etc.) in compressed concrete and reinforcement, Figure 4.

The total bending moments with cracks from small squares in compressed and stretched zones has the form:

$M_{\text {bend, sum }}=v_{b}(\lambda) \cdot E_{b} \cdot A_{b} \cdot z_{b} \iiint f_{\text {sum }, \Delta-d} d x d y d z+$

$+\sum_{\substack{k=1 \\ i=1}}^{n, k}\left(\sigma_{s, m, i, k} \cdot A_{s, i, k} \cdot z_{s, i, k}\right)=\sum_{i=1}^{m}\left(\sigma_{b, x, u} \cdot \omega_{\sigma, i} \cdot A_{b, c, i} \cdot z_{b, i}\right)+$

$\sum_{i=1}^{n-m}\left(\sigma_{s, r i g, m, i} \cdot A_{s, r i g, i} \cdot z_{s, i}+\sigma_{s, l e f, m, i} \cdot A_{s, l e f, i} \cdot z_{s, i}\right)+$

$+\sum_{i=1}^{m}\left(\sigma_{s^{\prime}, \text { ring, } m, i} \cdot A_{s^{\prime}, r i g, i} \cdot z_{s^{\prime}, i}+\sigma_{s^{\prime}, l e f, m, i} \cdot A_{s^{\prime}, l e f, i} \cdot z_{s^{\prime}, i}\right)+$

$\sum_{j=1}^{j}\left(q_{s w, r i g}^{*} \cdot\left(a_{j}-c_{j}\right) \pm q_{s w, l e f}^{*} \cdot\left(a_{j}-c_{j}\right)\right)$

977
Where $\mathrm{n}$ - total number of small squares; $\mathrm{m}$ - the number of squares of the compressed area longitudinal reinforcement; $\mathrm{k}$ - transverse reinforcement with normal cracks and lateral cracks; j - cross-sections 1-6; $\omega_{\sigma}-$ filling area for stress diagram.

The total torque with cracks has the form:

$M_{t, \text { sum }}=Y_{2} \cdot \varphi_{A, i}(z, y) \cdot f_{5, \ldots, j]}(z, y)+$

$+\sum_{\substack{k=1 \\ i=1}}^{n, k}\left(\sigma_{s, m, i, k} \cdot A_{s, i, k} \cdot z_{s, i, k}\right)=$

$=\sum_{i=1}^{m}\left(\gamma_{t, b, \text { sum }, u} \cdot v_{b}(\lambda) \cdot \omega_{\gamma, i} \cdot A_{b, c, i} \cdot z_{\eta, b, i}\right)+$

$+\sum_{i=1}^{n-m}\left(\sigma_{s, r i g, m, i} \cdot A_{s, r i g, i} \cdot b_{s, i}+\sigma_{s, l e f, m, i} \cdot A_{s, l e f, i} \cdot b_{s, i}\right)+$

$+\sum_{i=1}^{m}\left(\sigma_{s^{\prime}, r i g, m, i} \cdot A_{s^{\prime}, r i g, i} \cdot b_{s^{\prime}, i}+\sigma_{s^{\prime}, l e f, m, i} \cdot A_{s^{\prime}, l e f, i} \cdot b_{s^{\prime}, i}\right)+$

$+\sum_{j=1}^{j}\left(q_{s w, r i g, i} \cdot\left(a_{j}-c_{j}\right) \cdot z_{\eta, i} \pm q_{s w, l e f, i} \cdot\left(a_{j}-c_{j}\right) \cdot z_{\eta, i, *}\right)+$

$+\sum_{j=1}^{j}\left(q_{s w, \sigma, r i g, i} \cdot\left(a_{j}-c_{j}\right) \cdot z_{\eta, \sigma, i} \pm q_{s w, \sigma, l e f} \cdot\left(a_{j}-c_{j}\right) \cdot z_{\eta, \sigma, i,{ }^{*}}\right)$

Where $\mathrm{n}$ - total number of small squares; $\mathrm{m}$ - the number of squares of the compressed area longitudinal re- 
inforcement; $\mathrm{k}$ - transverse reinforcement with normal cracks and lateral cracks; j - cross-sections 1-6; $Y_{2}-$ see (35); $\varphi_{\mathrm{A}, \mathrm{i}}(\mathrm{z}, \mathrm{y})$ - see (36); $\omega_{\mathrm{y}, \mathrm{i}}$ - filling area for diagram of shear deformations; $z_{\eta, b, i}, b_{s, i}, b_{s^{\prime}, i}, z_{\eta, \sigma, i}, z_{\eta, \sigma, i, *}-$ distance from point $\mathrm{O}^{*}$ to any point.

\section{CONCLUSIONS}

1. A simple method from a family of mesh methods was found for developing linear and angular deformation functionals by approximating rectangular sections in compressed and stretched regions.

2. We analyzed diagrams of angular deformations and shear stresses, defined functionals, obtained a new hypothesis of angular deformations and corollaries from the hypothesis.

3. The bending and torque moments were presented using new functionals, the projection coefficients of normal and tangential stresses were determined using diagrams of compressed concrete.

4. The areas of filling of the diagram $\omega_{\varepsilon}(x, y, z)\left(\omega_{\sigma}(x-\right.$ $, y, z))$ and $\omega_{y}(x, y, z)\left(\omega_{T}(x, y, z)\right)$ were obtained from the functionals of the bending and torque moments.

5. The analysis of the new functionality and functions of Timoshenko-Goodyer has been carried out. The error in finding the value of the functional considered is $2 \%$ at the points considered and $7 \%$ at any points of the cross section.

6. The total bending moments and torque are obtained in simple expressions and in full form.

\section{ACKNOWLEDGEMENT}

The work described in this paper has been conducted in the South-West State University of Russia.

\section{REFERENCES}

1. Lessing N.N. (1959). Determination of the bearing capacity of reinforced concrete elements of rectangular cross-section, working in bending with torsion. Investigation of the strength of elements of reinforced concrete structures, vol. 5, 3-28.

2. Lessig N.N., Rullay L.K. (1972). General principles for calculating the torsional flexural strength of reinforced concrete bars. Theory of reinforced concrete dedicated to the 75th anniversary of the birth of A.A. Grozdev, 43-49.

3. Zalesov A.S., Khozyainov B.P. (1991). Strength of reinforced concrete elements in torsion and bending. Proceedings of universities in chapter Construction and architecture, no. 1, 1-4.

4. Arzamastsev S.A., Rodevich V.V. (2015). To the calculation of reinforced concrete elements for bending with torsion. Proceedings of higher educational institutions. Building, vol. 681. no. 9, 99-109.
5. Ilker Kalkan, Saruhan Kartal. (2017). Torsional Rigidities of Reinforced Concrete Beams Subjected to Elastic Lateral Torsional Buckling. International Journal of Civil and Environmental Engineering, vol. 11, no.7, 969-972.

6. G. Klein, G. Lucier, S. Rizkalla, P. Zia and H. Gleich. (2013) Torsion simplified: a failure plane model for design of spandrel beams. ACI Concrete International Journal, 1-19.

7. Karpenko N.I. (1970). Determination of deformations of rod-shaped reinforced concrete box-shaped elements with torsional cracks. Cross-sectoral construction issues. "Domestic Experience", no. 10, 39-42.

8. Karpenko N.I., Elagin E.G. (1970). Deformations of reinforced concrete tubular elements subjected to torsion after cracking. Concrete and reinforced concrete, no. 3, 3-12.

9. Karpenko N.I. (1972). To the calculation of deformations of reinforced concrete rods with cracks in bending with torsion. Theory of reinforced concrete dedicated to the 75th anniversary of the birth of A.A. Gvozdev, 50-59.

10. Karpenko N.I. (1976). The theory of deformation of reinforced concrete with cracks. Stroyizdat, Moscow.

11. Karpenko N.I. (1996). General models of reinforced concrete mechanics. Stroyizdat, Moscow.

12. Travush V.I., Karpenko N.I., Kolchunov V.I., Kaprielov S.S., Demyanov A.I., Konorev A.V. (2018) The results of experimental studies of structures square and box sections in torsion with bending. Building and Reconstruction, vol. 80, no. 6, 32-43.

13. Travush V.I., Karpenko N.I., Kolchunov VI. I., Kaprielov S.S., Demyanov A.I., Bulkin S.A., Moskovtseva V.S. (2020). Results of experimental studies of high-strength fiber reinforced concrete beams with round cross-sections under combined bending and torsion. Structural mechanics of engineering structures and structures, vol. 16, no. 4, 290-297, DOI:10.22363/1815-5235-2020-16-4-290-297.

14. Travush V.I., Karpenko N.I., Kolchunov VI. I., Kaprielov S.S., Demyanov A.I., Konorev A.V. (2019). Main results of experimental studies of reinforced concrete structures of high-strength concrete b100 round and circular cross sections in torsion with bending. Structural Mechanics of Engineering Constructions and Buildings, vol. 15, no. 1, 51-61. DOI:10.22363/18155235-2019-15-1-51-61

15. Demyanov A.I., Salnikov A.S., Kolchunov VI. I. (2017). The experimental studies of reinforced concrete constructions in torsion with bending and the analysis of their results. Building and Reconstruction, vol. 72, no. 4, 17-26. 
16. Demyanov A.I., Kolchunov V.I., Pokusaev A.A. (2017). Experimental studies of the deformation of reinforced concrete structures during torsion with bending. Structural Mechanics of Engineering Constructions and Buildings, no. 6, 37-44, DOI:10.22363/1815-5235-2017-6-37-44

17. Kolchunov V.I., Kolchunov VI. I., Fedorova N.V. (2018). Deformation models of reinforced concrete under special impacts. Industrial and civil construction, no. 8, 54-60.

18. Kolchunov VI. I., Fedorov V.S. (2020). Conceptual Hierarchy of Models in the Theory of Resistance of Building Structures. Industrial and Civil Engineering, no. 8, 16-23, DOI: 10.33622/0869-7019.2020.08.16-23.

19. Fedorov, V. S., Kolchunov VI. I., Pokusaev A.A., Naumov N.V. (2019). Design models of deformation of reinforced concrete structures with spatial cracks. Scientific journal of construction and architecture, vol. 56 , no. $4,11-28$.
20. Bondarenko V.M., Kolchunov V.I. (2004). Design models of the power resistance of reinforced concrete. Publishing house ABC, Moscow.

21. Velyuzhsky Yu.V., Golyshev A.B., Kolchunov VI.I., Klyueva N.V., Lisitsin B.M., Mashkov I.L., Yakovenko I.A. (2014). A reference guide to structural mechanics: Volume II. Publishing house ABC, Moscow.

22. Kolchunov, V. I., Dem'yanov A. I. (2019) The modeling method of discrete cracks and rigidity in reinforced concrete. Magazine of Civil Engineering, vol. 88, no. 4, 60-69, DOI: 10.18720/MCE.88.6.

23. Karpenko, N. I., Kolchunov VI. I., Travush V. I. (2021) Calculation model of a complex stress reinforced concrete element of a boxed section during torsion with bending. Russian Journal of Building Construction and Architecture, vol. 51, no. 3, 7-26, DOI: 10.36622/VSTU.2021.51.3.001.

24. Timoshenko S.P., Goodyer J. (1975). Theory of elasticity. Nauka, Moscow. 